\title{
Justicia global y seguridad humana en el contexto del cambio climático
}

\author{
Global Justice and Human Security in the Context \\ of Climate Change
}

\author{
Miguel MoRenO MuÑOZ \\ Universidad de Granada \\ mm3@ugr.es
}

RESUMEN. El concepto de justicia global ha permitido articular formas diversas de reacción al globalismo del mercado. Aunque muchos de sus desafíos siguen vigentes, han adquirido nuevos significados en el contexto de la crisis económica, social y humanitaria inducida por los fenómenos extremos asociados al cambio climático. Los conceptos de democracia y justicia, teorizados habitualmente en contextos nacionales, pueden ocasionar ciertas disfunciones cuando se aplican a contextos de problemas trans-nacionales que pueden comprometer la seguridad de individuos y comunidades, como son los asociados al cambio climático. Un enfoque centrado en la noción de seguridad humana obliga a considerar aspectos cruciales de la relación entre crisis ambiental, desarrollo no sostenible y riesgo de deriva autoritarista o conflicto violento, con implicaciones sustantivas para el concepto de justicia global y los mecanismos democráticos de gobernanza internacional.

Palabras clave: justicia global, seguridad humana, cambio climático, autoritarismo, conflicto.
ABSTRACT. The concept of global justice has inspired diverse reactions to market globalism. Although many of its challenges are still in force, they have acquired new meanings in the context of the economic, social and humanitarian crisis induced by the extreme phenomena associated with climate change. The constructs of democracy and justice, usually theorized in national contexts, may fail to function properly when applied to transnational contexts of problems, which can compromise the safety of individuals and communities (like those associated with climate change). A new frame, centred on the notion of human safety, is needed to consider crucial aspects in the relation between environmental crisis, unsustainable development and risk of authoritarian bias or violent conflict, with substantive implications for the concept of global justice and the democratic mechanisms of international governance.

Key words: global justice, human security, climate change, authoritarianism, conflict. 


\section{Introducción}

El informe del Foro Humanitario Global, Climate Change - the Anatomy of a Silent Crisis (GHG, 2009), muestra de forma inequívoca la estrecha asociación entre pobreza y vulnerabilidad extrema al cambio climático. El aumento de apenas un grado centígrado en la temperatura media global podría provocar hambrunas, migraciones en masa y amenazas a la salud pública en diversas partes del mundo. Pero los efectos globales del cambio climático son especialmente dañinos para los sectores más desfavorecidos de la población mundial, precisamente los que menos han contribuido al calentamiento global. Esto plantea cuestiones complejas en materia de justicia distributiva y equidad ambiental, afectando a los intereses reales de generaciones presentes y futuras. Por tanto, una primera sección de este trabajo se dedica a analizar los elementos de intersección entre los conceptos de justicia global y justicia ambiental, con el propósito de identificar criterios de gobernanza internacional útiles para enfocar la interacción Norte-Sur en lo relativo a la gestión de los efectos del cambio climático.

Se analizan a continuación los efectos del cambio climático en el deterioro de las condiciones de vida en los países más pobres y con sistemas de gobierno más frágiles, destacando sus implicaciones en términos de seguridad humana. Un número creciente de informes y análisis geopolíticos de relevancia internacional coinciden en destacar los riesgos de conflicto violento y deriva autoritaria ligados al deterioro de la economía, la salud, el acceso al agua y la escasez de recursos básicos para el mantenimiento de las condiciones y medios de vida, como consecuencia de los fenómenos meteorológicos extremos asociados al cambio climático. Si bien esta asociación necesi- ta mayor investigación empírica, existen numerosos precedentes a tener en cuenta y se están produciendo episodios concomitantes que responden a las previsiones menos optimistas avanzadas por el grupo de expertos del IPCC (Intergovernmental Panel on Climate Change) en 2007.

La incorporación de la perspectiva de seguridad humana, en términos clásicos o a través del análisis de modelos y pautas culturales de desarrollo que incrementan la vulnerabilidad de individuos, comunidades y países, contribuye a reforzar el concepto de justicia global en el complejo marco de riesgos y oportunidades suscitado por los impactos físicos y humanos del cambio climático. Su contribución al desarrollo de mecanismos democráticos de gobernanza internacional para una gestión eficaz de la crisis ambiental puede ser decisiva, siempre que sus aplicaciones y desarrollos sean compatibles con las exigencias de responsabilidad común y diferenciada que la noción de justicia climática ayuda a dilucidar.

\section{Justicia global y justicia climática}

La ciudadanía de los países menos desarrollados carece de la capacidad para hacer oír sus voces en los foros internacionales; pero el cambio climático supone una amenaza generalizada y directa para el medio natural, la economía, la salud y la seguridad, que no discrimina en sus efectos a colectivos bien o mal representados en las instituciones internacionales. Este fenómeno complejo pone a muchas comunidades bajo presión extrema, siendo las más expuestas a padecer sus efectos precisamente aquéllas que menos responsabilidad tienen en las emisiones de gases de efecto invernadero. Se asume, en consecuencia, que los criterios de justicia y equidad deben sustentar el marco conceptual necesario para afrontar la 
problemática compleja del cambio climático.

El análisis pormenorizado de los efectos inmediatos y previsibles del cambio climático ha reforzado extraordinariamente la dimensión ecológica y ambiental de todos los constructos teóricos sobre la justicia, hasta el punto de que muchos contenidos y exigencias fundamentales ligadas al concepto de «justicia global» quedan subsumidos hoy en la noción de justicia ambiental o justicia climática, de creciente aceptación tras la formulación propuesta en la Cumbre de Bali de 2002. ${ }^{1}$

El concepto de justicia global cercó un amplio terreno ideológico con creciente visibilidad — a partir de 2001gracias al Foro Social Mundial. Desde entonces ha permitido articular formas diversas de reacción al globalismo del mercado, aunque su aceptación haya sido muy dispar. Sin embargo, muchos de sus desafíos siguen vigentes y han adquirido nuevos significados en el contexto de la crisis económica, social y humanitaria inducida por los fenómenos extremos asociados al cambio climático.

En relación con los fenómenos migratorios, por ejemplo, el concepto de justicia global ha servido de contrapeso a las iniciativas políticas más restrictivas en el control de fronteras que establecían, de hecho, barreras insalvables para que millones de ciudadanos de los países menos desarrollados tuviesen opciones de mejorar sus condiciones de vida y las de sus allegados. La facilidad con que estas restricciones podían ampararse en razones de presunta sostenibilidad económica o social del modelo de desarrollo predominante en los países de acogida queda en entredicho cuando, bajo la perspectiva ambiental, un elevado porcentaje de esta población se ve obligada a desplazarse por el impacto del cambio climático —un fenómeno al que apenas han contribuido- en su modo de vida, en el acceso a recursos básicos como el agua y en el empeoramiento de parámetros de salud básicos.

Los refugiados ambientales constituyen una categoría relativamente nueva para el marco de garantías jurídicas que, en la esfera internacional, habría de concretar los derechos de un colectivo muy numeroso y creciente de desplazados (las estimaciones oscilan entre 25 y 135 millones de desplazados hasta 2020; para 2050 podrían superar los 250 millones). Cuanto más se difumina la línea divisoria entre migración forzosa y voluntaria, más fuerza adquiere la conexión entre las exigencias de justicia global y el impacto ambiental de los diversos modelos de desarrollo. No obstante, el estatuto jurídico internacional de los refugiados - recogido en la Convención de Ginebra de 1951 - no contempla la figura del «refugiado ambiental». Incluso reconociendo que, muy probablemente, las causas ambientales están detrás de un mayor número de personas forzadas a desplazarse que las guerras, el Alto Comisionado de las Naciones Unidas para los Refugiados (ACNUR) y otras organizaciones prefieren hablar de «personas ambientalmente desplazadas», en su propio país o a través de fronteras internacionales, debido al deterioro irreversible del medio ambiente o al aumento del nivel del mar (Borràs, 2006).

El problema de los refugiados ambientales, y su exclusión como categoría de sujetos que merecen una protección jurídica por razones específicas, ilustra bien las limitaciones de un marco de derechos que no responde de manera satisfactoria a demandas genuinas de justicia global. Esto explica que algunos autores hayan optado por el concepto de «justicia climática» como noción preferible para abordar desafíos concretos en la problemática ambiental que, bajo una perspectiva genérica de justicia global, podrían 
quedar desatendidos, a saber: ampliar el concepto de refugiado; establecer reparaciones y responsabilidades judiciales, civiles o penales ante intervenciones que conllevan la destrucción o deterioro irreversible del entorno natural; la creación de fondos de compensación; la puesta en marcha de estrategias de adaptación y mitigación para frenar el impacto de la actividad humana en el clima, etc.

A diferencia de otros constructos teóricos sobre la justicia, J. Goodman (2009) considera que la noción de justicia climática aporta una trayectoria bien definida en aspectos cruciales (alcance, discurso, espacio, estrategia, temporalidad y agencia), con sus propios dilemas y potenciales. Aduce que la justicia climática es un concepto totalizador, científicamente mensurable, que aporta el nuevo impulso que necesitan los países de industrialización tardía, que exige estrategias proactivas en un horizonte temporal limitado y en el marco de una epistemología de gran alcance, radicalmente desafiante.

Estos aspectos de la justicia climática apuntalan con firmeza elementos más dispersos en las nociones de justicia ecológica o justicia ambiental y permiten abordar algunas limitaciones de la justicia global, aunque plantean sus propios problemas. Pero Goodman considera la dinámica emergente de la justicia climática el detonante hacia una «transición paradigmática», capaz de impulsar transformaciones a gran escala en términos de contestación política.

Siendo relevantes todos los matices diferenciadores, parece poco operativo manejar varios conceptos de justicia con zonas amplias de intersección en los foros donde las decisiones, si llegan, surgen de rondas de negociación complejas. A tal efecto, la noción de justicia climática parece susceptible de incorporar las exigencias normativas aplicables a la dimen- sión humana y natural de los impactos del cambio climático, de manera compatible con los criterios establecidos de responsabilidad común y diferenciada, y de integrar tanto la dimensión teórica vinculada a las interpretaciones del conocimiento científico disponible como la dimensión práctica de acción y contestación política ante las reticencias de los estados a implicarse activamente en las soluciones. Mientras que la noción de justicia global se asocia inequívocamente con las exigencias de justicia trans-fronterizas, en un contexto de creciente interdependencia económica, social y política de los estados-nación, muchos de los cuales son incapaces de hacer frente con sus propios recursos a las amenazas del calentamiento global para la seguridad, la salud y la economía de sus comunidades.

La magnitud de los desafíos a los que se enfrentan los estados nación como consecuencia del impacto inmediato y previsible del cambio climático ha puesto de manifiesto las limitaciones e insuficiencias flagrantes del marco actual de principios en el que se desarrollan las negociaciones y acuerdos de adaptación y mitigación. La sucesión concomitante de fenómenos meteorológicos extremos en grandes zonas de todo el planeta evidencia la escasa operatividad de los enfoques centrados en el protagonismo exclusivo de los estados y sus intereses. Unas lluvias anormalmente intensas y persistentes bastan para llevar al colapso todas las estructuras estatales existentes en grandes zonas del planeta y alterar de modo irreversible y dramático las condiciones de vida de decenas de millones de personas, al margen de su nacionalidad. Es preciso, en consecuencia, redimensionar el alcance del concepto de justicia global en el nuevo contexto de riesgos climáticos supra-estatales y las implicaciones de la noción de justicia ambiental en coherencia con criterios de gobernanza democrática internacional. 


\section{Complejidad y dimensión global de los problemas ambientales}

El informe del IPCC de 2007 confirma con mayor respaldo empírico lo que ya señalaba otro informe del mismo organismo en 2001: «el calentamiento del sistema climático es inequívoco» $\mathrm{y}$, durante el último medio siglo, «muy probablemente se debe al aumento observado en las concentraciones antropogénicas [de los gases de efecto invernadero]» (IPCC, 2007a, 1, 4). Las proyecciones, sobre diversos escenarios, coinciden en asociar dicho fenómeno con una serie de efectos potencialmente dañinos a escala global pero que, en algunas regiones del planeta, pueden ser repentinos, irreversibles y devastadores para los sistemas humanos y naturales (IPCC, 2007b). Por lo tanto, el cambio climático representa una amenaza que puede alterar de manera muy significativa el entorno natural, la economía, la salud y la seguridad de muchas comunidades humanas.

Los países en desarrollo soportan un 90\% de las pérdidas económicas totales, representan el $98 \%$ de los más gravemente afectados y en ellos se produce el $99 \%$ de las muertes por catástrofes asociadas a una climatología adversa. Los 50 países menos desarrollados contribuyen menos del $1 \%$ a las emisiones totales de $\mathrm{CO}_{2}$, pero es en ellos donde el clima puede acentuar las desigualdades ya existentes entre mujeres, niños y personas mayores, los grupos más vulnerables (GHF, 2009).

Dentro de las comunidades, las consecuencias del cambio climático y la pobreza no tienen una distribución uniforme. Factores individuales y sociales determinan la vulnerabilidad y la capacidad para adaptarse a los efectos del cambio climático. Las mujeres constituyen dos terceras partes de los pobres del mundo y siete de cada diez trabajadores agrícolas. Mujeres y niños están representados desproporcionadamente entre los desplazados por fenómenos meteorológicos extremos y otros efectos del clima. Los más pobres son los más duramente golpeados; pero el impacto humano del cambio climático es una cuestión global.

El coste humano de las olas de calor recientes, inundaciones, tormentas y fuegos forestales en países ricos está siendo alarmante (Doherty et al., 2009). Australia es quizás la nación desarrollada más vulnerable a los impactos directos del cambio climático, y también al impacto indirecto de los países vecinos afectados por el cambio climático. Pero Rusia, Estados Unidos, Canadá, Francia y otras naciones desarrolladas están siendo severamente afectadas, y muy probablemente lo serán en mayor proporción (Pengelly et al., 2007; Keatinge, Donaldson, 2004).

Las migraciones de personas desplazadas de zonas recientemente convertidas en inhabitables plantea un problema de seguridad de gran alcance, cuya respuesta es motivo de seria preocupación para la ONU. ${ }^{2}$ Las previsiones de disminución de los recursos hídricos en el sur de Asia y África afectarán a países pobres y ricos; pero en África será motivo de hambrunas y desplazamientos humanos. La escasez de agua potable en Asia, Iberoamérica y Europa por la disminución de los glaciares puede repercutir en una reducción significativa de la capacidad de producción de alimentos en las regiones más pobres, generando hambre y miseria si, como es previsible, afecta a cultivos básicos (trigo, maíz, y arroz en China e India).

Los problemas derivados de la escasez de agua potable pueden agravarse si, conforme a las estimaciones para incrementos de un grado centígrado en la temperatura media global, se produce una subida significativa del nivel del mar y aumenta la frecuencia de huracanes e inundaciones de los grandes deltas asiáticos (GHF, 2009). 
Las nuevas estrategias políticas en relación con el clima no pueden desatender exigencias básicas de justicia ambiental. Deben contribuir a empoderar a las comunidades vulnerables y a armonizar los valores democráticos básicos (participación, deliberación, etc.) con la eficacia de las medidas supraestatales acordadas para afrontar los desafíos del calentamiento global.

A escala internacional, se echa en falta una estrategia concertada y mucho más ambiciosa en lo relativo a objetivos de reducción de emisiones; pero, sobre todo, se precisa un nuevo marco que permita a cada país asumir sus propios objetivos y responsabilidades, sobre la base de un conocimiento compartido de los riesgos, del carácter urgente de las acciones a emprender y de la necesidad de colaboración trans-fronteriza en materia de mitigación y adaptación al cambio climático (Hepburn, Stern 2008: 259).

Bajo esta perspectiva pronto resulta evidente que los conceptos de democracia y justicia, teorizados habitualmente en contextos nacionales, originan serias disfunciones cuando se aplican a contextos de problemas trans-nacionales. Las limitaciones de la teoría de Rawls, del «liberalismo verde» y de los enfoques cosmopolitas, en general, para abordar la cuestión ambiental conforme al nivel de complejidad que el conocimiento disponible hoy demanda han sido señaladas por varios autores (Valdivielso, 2004a, 2004b; Maltais, 2008; Wissenburg, 2006). Los escollos fundamentales surgen en relación con la ampliación del concepto de «comunidad» (para incluir los intereses de las generaciones futuras o de otras especies) y por la dificultad para establecer mecanismos que permitan a los individuos asumir plena responsabilidad sobre sus impactos en el medio natural.

Muchas teorías anti-cosmopolitas han sido desarrolladas o sugeridas por los propios liberales. La concepción política de la justicia representa uno de los desafíos más importantes al cosmopolitismo en general, y un serio desafío para considerar los problemas ambientales globales en términos de justicia cosmopolita. Cuestiones como la contaminación del aire a largo plazo, el agotamiento de recursos ocasionado por los sistemas internacionales de producción y la dependencia de toda la humanidad de una biosfera compartida, son ejemplos de problemas ambientales trans-fronterizos que demandan una acción política concertada (Lidskog, Elander 2009). Aunque este tipo de asuntos se suelen tratar mediante negociaciones entre estados-nación, en el caso del cambio climático se plantean serias dudas acerca de la conveniencia de un enfoque similar:

Las naciones-Estado pobres temen que los acuerdos internacionales limiten sus pretensiones de crecimiento económico, mientras que las naciones-estado económicamente pujantes se niegan a reducir sustancialmente sus emisiones de gases de efecto invernadero (GHG) si los países en vías de desarrollo no hacen un sacrificio similar. Por lo tanto, el cambio climático involucra aspectos fundamentales de justicia global que constituyen auténticos atolladeros para las iniciativas políticas relacionadas con el cambio climático. [...] El desafío que supone definir y desarrollar estructuras para la acción política más allá del estado nación — que permitan afrontar las grandes cuestiones en materia de desigualdades globales y justicia ambiental- es un objetivo que dista mucho de haberse abordado con éxito (Lidskog, Elander 2009: 2-3. Trad. mía).

Se cuestiona, por lo tanto, que la democracia representativa, en la configuración territorialmente circunscrita del estado-nación, resulte útil para gestionar con eficacia problemas trans-fronterizos de naturaleza compleja y sujetos a debate intenso (Held, Koenig-Archibugi 2005). Si el sistema político fracasa en el desa- 
rrollo de instrumentos que permitan afrontar la cuestión del clima por vías democráticas, es muy probable que su función termine siendo reemplazada por los dictámenes de científicos y tecnócratas, como única respuesta eficaz a este desafío.

\section{Justicia climática y gobernanza democrática}

Afrontar el cambio climático de manera compatible con criterios democráticamente aceptables exige conocer en profundidad el potencial y las limitaciones que presenta el sistema emergente de gobernanza multinivel. Lidskog y Elander identifican cuatro desafíos que la ecología plantea a la democracia: (1) justicia entre diferentes regiones del mundo; (2) justicia inter-generacional; (3) el valor de las especies no humanas; y (4) la valoración del papel del conocimiento científico y de la competencia técnica en la toma de decisiones. Sostienen que debe preservarse la funcionalidad de los tres mecanismos democráticos fundamentales - representación, participación y deliberación- en las plataformas donde se dirimen posibles vías de respuesta al cambio climático, de modo que el resultado final no termine amparando, por razones de presunta eficacia, iniciativas que menoscaben los valores básicos de la democracia.

La falta de coherencia entre los diversos conceptos vigentes de justicia ambiental puede degenerar en un bucle de teorización caótica e interminable, sin efecto apreciable para suscitar consenso. Según Ikeme (2003), como constructo teórico la justicia ambiental tiene dimensiones distributivas y procedimentales, puede ser racionalizado con argumentos consecuencialistas o deontologistas, y puede ser diseccionado bajo perspectivas preventivas, correctivas o retributivas.
Este autor identifica tres grandes cuestiones de justicia ambiental y equidad de cara al debate sobre cambio climático: distribución de impactos, distribución de responsabilidad y distribución de costes y beneficios. Sin embargo, Norte y Sur afrontan el problema adoptando concepciones muy diferentes de equidad y justicia ambiental. El Sur suele priorizar la igualdad y destacar tanto las injusticias distributivas como los elementos correctores derivados de las emisiones históricas. El Norte se centra principalmente en las vías más eficientes, desde el punto de vista económico, para minimizar el impacto climático y distribuir, a escala global, los beneficios derivados de un entorno ecológicamente estable y saludable.

El resultado es que Norte y Sur, por lo general, se adhieren a fórmulas opuestas para el reparto de responsabilidades y cargas en la cuestión ambiental. Estas concepciones incompletas y, en muchos aspectos, contrapuestas de la justicia señalan la línea divisoria en la política Norte-Sur respecto del clima (Ikeme, 2003: 200).

Para incrementar su participación en el proceso de respuesta al cambio climático, los representantes de países del Sur aducen que la justicia o imparcialidad de un resultado dependen de la legitimidad del proceso donde se obtiene. Según Rawls (1972), una negociación justa siempre produciría un resultado justo, pero requiere una participación lo más amplia posible. La exigencia de equidad en la justicia procedimental establece que la distribución de costes y beneficios de los recursos atmosféricos sólo puede ser equitativa si surge de un proceso acordado por todas las partes. Sin embargo, hasta el momento, las negociaciones sobre el clima han sido mucho más sobre la protección de los intereses nacionales que sobre la protección del medio ambiente mundial. 
Pese a todo, tanto las posiciones morales consecuencialistas como las deontologistas adoptadas por el Norte y el Sur convergen en una misma conclusión: las mayores cargas para la protección del clima deberían recaer sobre el Norte, y la transferencia de recursos y tecnología de Norte a Sur debería utilizarse para facilitar la protección del clima y las medidas de adaptación en el Sur. Esto queda muy lejos de la posición de Rawls, para quien los pueblos de culturas diferentes no poseen un sustrato cultural (no-político) común para 'construir' un orden o sistema cosmopolita justo.

Motivados precisamente por cuestiones de desarrollo en el Sur, Amartya Sen y Martha Nussbaum han intentado construir una filosofía universalista de las «capacidades humanas» que podría considerarse un término medio entre Rawls y el cosmopolitismo de Pogge, Singer y Beitz (Doyle, 2006: 119). Aunque excede los objetivos de este trabajo, el análisis de algunos aspectos de la pobreza desde un enfoque centrado en las capacidades permite identificar aspectos de la seguridad humana que merecen una consideración detenida en materia de criterios de desarrollo en contexto de cambio climático (Heyd, Brooks, 2009).

Una definición precisa de seguridad humana apunta a las condiciones en las cuales los seres humanos y las comunidades tienen la capacidad de hacer frente a las amenazas o tensiones sobre sus necesidades, derechos y valores. Sabina Alkire (2003) propone entender la seguridad humana como "proteger el núcleo vital de todas las vidas humanas de las amenazas críticas generalizadas, de manera compatible con la satisfacción de los fines humanos a largo plazo».

El énfasis en la capacidad y condiciones para ejercer estas opciones sobre aspectos básicos de la vida es aún mayor en la propuesta del GECHS (Global
Environmental and Human Security Change), del International Human Dimensions Programme (IHDP), donde la seguridad humana se define como «un estado que se alcanza cuando y donde los individuos y las comunidades tienen las opciones necesarias para poner fin, mitigar o adaptarse a las amenazas a sus derechos humanos, ambientales y sociales; tienen la capacidad y la libertad de ejercer estas opciones, y de participar activamente en la consecución de estas opciones» (GECHS, 2009). ${ }^{3}$

\section{Impacto humano del cambio climático $y$ amenazas para la seguridad}

El análisis pormenorizado de los fenómenos meteorológicos extremos recientes en Rusia, Pakistán, Sahel y otras partes del planeta sugiere que su intensidad y frecuencia es consistente con las proyecciones contenidas en el informe de 2007 del IPCC sobre el impacto del cambio climático. En un comunicado reciente, la Organización Meteorológica Mundial (OMM) considera «sin precedentes» fenómenos concomitantes extremos como la ola de calor en Rusia, las inundaciones en vastas extensiones de Asia y otras zonas de Europa central, la sequía en África subsahariana y los deslizamientos de tierra en China. ${ }^{4}$

Aunque resulta arduo determinar la relación entre un fenómeno extremo particular y el cambio climático, los datos disponibles del conjunto del sistema climático se ajustan a la tendencia que, sobre diversos escenarios, esbozaron los expertos del IPCC. En esta perspectiva, no resultan sorprendentes las desviaciones significativas al alza de las temperaturas máximas en zonas pobladas de EE.UU. - aunque las nevadas o lluvias del invierno pudieran sugerir lo contario-, donde el primer semestre del año fue el más cálido de los últimos 131 años. 
Roshydromet, el Servicio Federal Ruso de Hidrometeorología, confirma que el mes de julio de 2010 en Moscú ha sido el más cálido en 130 años — desde el inicio de los registros-, con una temperatura media 7,8 grados centígrados superior a la normal. Este incremento ha provocado múltiples incendios - muy difíciles de sofocar, porque en muchos casos es la turba acumulada en el subsuelo lo que se quema-y amenazas serias para la vida de personas, infraestructuras e instalaciones, incluidas las militares.

En «los peores incendios de su historia», según el Ministerio de Protección Civil de Rusia, el fuego, los fuertes vientos y los meses de sequía prolongada han destrozado más de 500 edificios de al menos 26 aldeas de las provincias de Volgogrado y Saratov; han dejado a miles de personas sin hogar y más de 17 heridos, que se suman a los 54 muertos por la ola de calor de julio-agosto. Los efectos del humo y la contaminación del aire están teniendo un impacto adverso importante en la salud y el bienestar de millones de personas. El coste económico de la ayuda necesaria para paliar los daños en las regiones de Volgogrado y Saratov asciende a más de 25,4 millones de euros. Y las primeras investigaciones muestran que las autoridades - dejando a un lado posibles casos de negligencia-, quedaron sobrepasadas por la magnitud de la emergencia. 5

La organización ecologista Greenpeace calcula los daños en más de 200.000 millones de euros y en al menos 12 millones las hectáreas quemadas, muy por encima del millón que reconocen las autoridades. La carencia de sistemas antifuego y de alerta puede traducirse en nuevos episodios de esta misma catástrofe, según Greenpeace. La sequía prolongada ya había malogrado gran parte de las cosechas y el presidente Putin anunció la posibilidad de prolongar la prohibición de exportar cereales más allá del 31 de diciembre. ${ }^{6}$

El Departamento de Meteorología de Paquistán (PMD) ha estado emitiendo alertas continuas y peticiones de asistencia en la ayuda de emergencia desde mediados de junio de 2010. Precipitaciones muy intensas y persistentes pasados provocaron en julio-agosto graves inundaciones, repentinas y muy extendidas por grandes zonas de todo el país. ${ }^{7}$ En la provincia de Khyber-Pakhtunkhwa, las lluvias caídas en julio representaban un $180 \%$ del promedio mensual a largo plazo. La población afectada roza los $15 \mathrm{mi}-$ llones de personas - superior a los afectados por el tsunami de 2004 o el terremoto de Haití-, y alrededor de 290.000 viviendas han resultado dañadas, según Naciones Unidas. ${ }^{8}$

Las 1.200 víctimas mortales directamente relacionadas con las inundaciones de Pakistán se consideran una cifra alta pero limitada, según la ONU, dada la dimensión de la catástrofe. El gran reto son los supervivientes, puesto que al menos 15 millones de personas están en riesgo y de ellos seis millones necesitan atención urgente, según John Holmes, responsable de la Respuesta de Emergencia. La escala del desastre y las necesidades son enormes, con dificultades insuperables para garantizar la seguridad alimentaria de la población vulnerable por la pérdida de cosechas y ganado, más las restricciones en el acceso al agua potable, que impiden atender necesidades sanitarias inmediatas. A los costes de la reconstrucción se suma la subida del precio de los cereales y de todos los alimentos, influido en parte porque la sequía y los incendios en Rusia forzaron un bloqueo temporal en las exportaciones de trigo que garantizara reservas suficientes para atender la demanda interna.

El rechazo a la ayuda internacional por parte de talibanes y algunas organiza- 
ciones caritativas islámicas como Jamaat-ud-Dawa puede obstaculizar considerablemente la colaboración en la asistencia a las víctimas de Pakistán. La ONU toma muy en serio las amenazas y cuenta con que algunos grupos armados pueden aprovechar la catástrofe en su beneficio y volver a atentar - como hicieron en 2009 en una oficina del Programa Mundial de Alimentos (PMA) en Islamabad, donde murieron cinco empleados de la agencia - contra los cooperantes internacionales. ${ }^{9}$

Estos ejemplos recientes y dramáticos de emergencias complejas ponen de manifiesto el carácter conservador de las proyecciones — realizadas en los últimos años a partir de modelos científicamente validados o de las mejores estimaciones disponibles - sobre el impacto humano del cambio climático. Y dan una idea aproximada de hasta qué punto puede verse comprometida la seguridad y la capacidad de resistencia de individuos y comunidades a escala planetaria (GHF 2009: 1-4).

Sólo los desastres derivados de fenómenos meteorológicos adversos están provocando pérdidas económicas que ascienden a 163.000 millones de euros en los últimos cinco años. Las evidencias disponibles indican que este tipo de desastres ha aumentado en frecuencia y gravedad en los últimos 30 años, en parte como consecuencia del cambio climático. A escala mundial es ya muy significativo - a escala local puede ser críticosu impacto sobre la salud, la producción de alimentos, el acceso al agua, la seguridad y las condiciones de vida de millones de personas.

Diversas organizaciones consideran que pueden quedar severamente comprometidos los Objetivos del Milenio en materia de desarrollo sostenible. Además, los mecanismos establecidos de asistencia humanitaria se verán sometidos a una presión importante y creciente, con estimaciones de más de 300.000 muertes anuales directamente atribuibles a sus efectos, más de 325 millones de personas seriamente afectadas, cerca de $4.000 \mathrm{mi}-$ llones en situación vulnerable, y alrededor de 500 millones de personas en situación de riesgo extremo. Las pérdidas económicas podrían llevar a la quiebra a las compañías de seguros más solventes (Harmon, 2009). Y los cientos de millones de personas afectadas hoy pueden ser más del doble en un plazo de 20 años, si se mantienen las tendencias actuales. Esto significaría que una de cada diez personas de la población actual se vería directa y seriamente afectada por el impacto del cambio climático, en una dimensión de la que no somos conscientes todavía (GHF, 2009: 2).

El impacto humano del cambio climático se está produciendo ya, y requiere atención urgente. Eventos como los desastres por climatología adversa, la desertificación y elevación del nivel del mar se están agravando por el cambio climático, afectando a individuos y comunidades de todo el mundo. Provocan hambre, enfermedad, pobreza y deterioro de los medios de vida, reducen el crecimiento económico y suponen una amenaza para la estabilidad social y política. Mucha gente es incapaz de hacer frente a los patrones climáticos extremos y su variabilidad. Son incapaces de proteger a sus familias, sus modos de vida y el suministro de alimentos ante los impactos negativos de las lluvias estacionales que provocan inundaciones o de la escasez de agua durante sequías prolongadas. El cambio climático está multiplicando estos riesgos (GHF, ibid.).

No se trata de una urgencia ficticia o exagerada: los expertos están revisando sus predicciones sobre el cambio climático, puesto que los datos disponibles indican que está ocurriendo más rápidamente de lo que sugerían hasta hace poco incluso los modelos más agresivos. El conocimiento disponible induce a pensar que el 
incremento de la frecuencia e intensidad de estos fenómenos, en una proporción significativa, guardan relación con la actividad humana, en particular con modelos de desarrollo intrínsecamente dependientes del incremento de las emisiones de gases de efecto invernadero $-\mathrm{CO}_{2}$ sobre todo, producto de la quema de combustibles fósiles-, que se han acumulado en la atmósfera en niveles sin precedentes durante cientos de miles de años y retienen parte del calor que emite la Tierra (Zeng et al., 2008). Y las concentraciones de estos gases en la atmósfera continúan en aumento (se estima un crecimiento del $56 \%$, desde las 27 giga-toneladas de 2005 hasta las 42 giga-toneladas hacia 2030) pese a los acuerdos internacionales sobre limitación de emisiones, con las implicaciones obvias para los fenómenos climáticos extremos asociados (Nature, 2007).

\section{Riesgo de conflicto violento}

Actualmente, más de quinientos millones de personas están en riesgo extremo ante las consecuencias del cambio climático, y seis de cada diez son vulnerables a sus efectos físicos y socioeconómicos. La mayoría de la población mundial no tiene la capacidad para enfrentarse a los impactos del cambio de clima sin sufrir una pérdida potencialmente irreversible de bienestar o poner en riesgo sus vidas. Las poblaciones en riesgo más grave e inminente viven en algunas de las áreas más pobres y más propensas a acusar los efectos del cambio climático - los países del cinturón semiárido desde el Sáhara hasta Oriente Medio y Asia Central, así como el África sub-Sahariana, las vías fluviales del Sur de Asia y las pequeñas islas-estado en desarrollo-. Las pérdidas económicas atribuibles al cambio climático superan los 70.000 millones de euros/año, cifra superior al PIB de 3/4 partes de los países del mundo, y más que toda la ayuda anual al desarrollo.

Se entiende, por tanto, que la relación entre cambio climático, seguridad humana y riesgo de conflictos violentos esté recibiendo una atención considerable, hasta el punto de que para muchos organismos e instituciones el cambio climático es ya, antes que otra cosa, un problema de seguridad (Barnett, Adger, 2007; Brauch, 2002; Barnett, 2003). Sus efectos pueden socavar los mecanismos de seguridad en vigor y someterlos a una presión creciente en el futuro, fundamentalmente restringiendo el acceso a, y la calidad de, los recursos naturales básicos para sustentar modos de vida. Por otra parte, es previsible que menoscabe de forma significativa la capacidad de los estados para seguir proporcionando oportunidades y servicios que permitan a la gente subsistir. Esta combinación de efectos directos e indirectos explica que el cambio climático pueda incrementar el riesgo de conflictos violentos.

Las amenazas para la seguridad pueden proceder de cambios en las políticas de recursos energéticos, por las directrices para reducir las emisiones de combustibles fósiles (Rifkin, 2002); pero también de las expectativas de conflicto, estimuladas por cambios en los sistemas sociales e inducidos éstos por impactos reales o percibidos del clima ( $P e w R e-$ search Center for the People and the Press, 2008). En este contexto, la seguridad humana puede resultar comprometida por individuos y comunidades que pierden la capacidad de gestionar con eficacia la presión que perciben sobre sus necesidades, derechos y valores (Alkire, 2003).

El tipo de inseguridad humana que el cambio climático puede provocar cuenta con numerosos precedentes que lo asocian (aunque no de manera determinante) con mayor riesgo de conflictos violentos. En muchos sentidos es claro que el cam- 
bio climático puede minar la capacidad de los Estados para promover la seguridad humana y la paz y - aunque esta asociación requiera más investigación empírica - por sus efectos directos en los modos de vida y los indirectos en las funciones de los estados, puede incrementar significativamente, en diversas circunstancias, el riesgo de conflicto violento.

Esta posibilidad ha sido tomada muy en serio por estados e instituciones internacionales al máximo nivel. El 17 de abril de 2007, el Consejo de Seguridad de Naciones Unidas dedicó una sesión específica a debatir el impacto del cambio climático en la paz y la seguridad. En ella, Ban Ki-Moon - Secretario General de Naciones Unidas - señalaba que a lo largo de la historia humana, pueblos y países habían luchado por los recursos naturales (ganado, pozos, tierra fértil, rutas comerciales, reservas de pesca, especias, azúcar, petróleo, oro y otras materias preciosas) y que muy a menudo ha sido la guerra el principal medio para asegurar la posesión de recursos escasos. Incluso hoy, el suministro ininterrumpido de combustible y minerales constituye un elemento clave de todas las consideraciones geopolíticas: "Cuando los recursos son escasos - se trate de energía, agua o tierra cultivable - nuestros ecosistemas frágiles se ven sometidos a una gran tensión, igual que los mecanismos de adaptación de individuos y grupos. [...] Esto puede conducir a una ruptura de los códigos establecidos de conducta, incluso al enfrentamiento directo.» ${ }^{10}$

Por otra parte, en diversos informes previos destinados a la prevención de conflictos, Kofi Annan - anterior Secretario General de Naciones Unidas - ya había advertido de las amenazas derivadas del deterioro ambiental y de la escasez de recursos, cuyo potencial para desestabilizar regiones ya de por sí proclives al conflicto se agrava cuando se combinan con desi- gualdad o politización en el acceso. Aunque las iniciativas orientadas a la prevención cuestan mucho menos que el conflicto y sus consecuencias, los desacuerdos en las medidas preventivas, en los plazos y en el régimen para repartir estos costes indican que están pesando menos las consideraciones sobre el valor y la calidad de las vidas humanas que otros intereses. Y esto es así incluso aunque formalmente la mayoría de los países reconoce que el cambio climático requiere una respuesta global a largo plazo, acorde a los nuevos datos científicos y compatible con formas sostenibles de desarrollo económico y social.

\section{Riesgo de deriva autoritaria}

La especulación sobre la relación entre problemas ambientales y regímenes autoritarios no se inaugura con la problemática del cambio climático; pero sí es probable que las dimensiones de los problemas y la severidad de los ajustes necesarios en este nuevo contexto renueven su actualidad (Wolf, 1999; Suhrke, 1997). Centrando su análisis en el sureste asiático, Beeson (2010) considera que los países con recursos estatales limitados lucharán como puedan para hacer frente a las consecuencias del crecimiento demográfico, el desarrollo económico y la degradación ambiental asociada. El resurgimiento de sistemas de gobierno autoritarios parece más probable en zonas geográficas donde existen ejemplos cercanos de desarrollo económico exitoso (en alusión expresa a China) y problemas ambientales graves de gran alcance. Aunque no resulten particularmente atractivos, algunos regímenes autoritarios pueden demostrar una capacidad mayor de respuesta a las presiones políticas y ambientales complejas que algunas democracias de la misma región.

A propósito del auge de las formas de capitalismo no liberales, Larry Dia- 
mond (2008) habla explícitamente de un fenómeno de «recesión democrática» de mayor alcance, y recuerda que no hay nada inexorable en el triunfo de los valores o prácticas políticas y económicas occidentales. Las prioridades de desarrollo entre las comunidades y gobiernos en las regiones más pobres del mundo podrían ser muy diferentes de las que tienen sus homólogos más opulentos en Europa y Norteamérica; es previsible que tengan también expectativas muy diferentes sobre las funciones que debe desempeñar un gobierno (Nisbet, Kotcher, 2009).

Por lo demás, una trayectoria de desarrollo económico y político percibida como exitosa pero ligada a regímenes frecuentemente autoritarios, puede contribuir a atrincherar estas alternativas cuando las disfunciones de los modelos no sostenibles de desarrollo sean patentes. En términos parecidos analiza Jared M. Diamond (2005) el riesgo de colapso social como consecuencia de la crisis ambiental y otras variables (relaciones con países vecinos, instituciones políticas, etc.). Y, partiendo de su análisis de las catástrofes naturales, Suzanne Leroy (2006) considera que el colapso social suele ser la consecuencia de una combinación de tiempo (la velocidad con que ciertos cambios en el clima o en la disponibilidad de ciertos recursos se producen), de localización geográfica (la extensión de terreno afectada por un fenómeno determinado) y del tipo de estructura social (rígida o flexible) desde la que se le hace frente.

Este riesgo no debería considerarse ficticio o meramente percibido, puesto que el problema ambiental se ha convertido en la cuestión determinante de la política pública hoy (Sinha, 2010; Roberts, Parks, 2007). Las respuestas políticas a los desafíos ambientales determinan la salud del planeta; pero una degradación ambiental persistente puede afectar tam- bién a los sistemas políticos. Una interacción que probablemente se agudizará en aquellas partes del mundo donde los problemas ambientales son más apremiantes y más débil la capacidad de los Estados para responder a ellos con eficacia.

Se trata de un contexto favorable para que las élites políticas se pongan de acuerdo en privilegiar el mantenimiento del régimen y la estabilidad interna frente a la liberalización política. Los esfuerzos para mitigar o adaptarse al impacto del cambio climático pueden implicar por sí solos una disminución en la libertad individual - y no sólo del nivel de vida - si los gobiernos deciden tomar medidas para modificar los hábitos y conductas ambientalmente nocivos.

Estas razones aconsejan evitar la precipitación a la hora de relegar conceptos de justicia que, aun necesitados de revisión y desarrollo, se han mostrado operativos en la contribución a los (todavía) débiles sistemas democráticos de gobernanza internacional.

\section{Conclusión}

El cambio climático exige una atención renovada a la dimensión ambiental de los diversos conceptos de justicia en uso. Los aspectos distributivos y las exigencias de equidad en el reparto de los costes de las estrategias de adaptación y mitigación suponen un desafío notable para los instrumentos disponibles de gobernanza internacional, centrados por lo general en los estados-nación y sus intereses. Puesto que los efectos del cambio climático van a afectar desproporcionadamente a las comunidades más pobres de los países menos industrializados, es preciso pensar los modelos de desarrollo en términos de seguridad humana, sea desde la perspectiva de las capacidades (que aquí se considera teóricamente más fructífera) o desde planteamientos alternativos. 
Es obvio que el surgimiento de una sociedad civil ecológica y políticamente sabia puede contribuir a modificar las prácticas ambientales ligadas a modelos de desarrollo injustos y desiguales, que terminan incrementando la vulnerabilidad de comunidades y países ante fenómenos climáticos extremos (Pattberg, 2007; Heyd, 2007). Pero esto es algo que también puede ser hecho, y con notable eficacia, desde estructuras políticas autoritarias. Una tendencia que cuenta con numerosos precedentes en zonas diferentes del mundo, y que puede contribuir a incrementar los riesgos de conflicto violento donde la escasez de recursos vitales comience a ser apremiante y las estructuras estatales demasiado débiles para garantizar servicios y oportunidades básicas.

El desafío para una noción operativa de justicia global estriba en incorporar con radicalidad las exigencias multidimensionales asociadas a los conceptos de justicia climática o ambiental y el potencial para consolidar logros irrenunciables en materia de seguridad, derechos y libertades asociados a las democracias liberales.

\section{BIBLIOGRAFÍA}

Alkire, S. (2003): A conceptual framework for human security, CRISE working paper 2, Oxford: Queen Elizabeth House.

BARnetT, J. Adger WN (2007): «Climate change, human security and violent conflict», Political Geography, 26: 639-655.

BARNETT, J. (2003): «Security and climate change», Global Environmental Change, 13: 7-17.

BEESON, M. (2010): «The coming of environmental authoritarianism», Environmental Politics, 19 (2): 276-294.
BORRÀs, S. (2006): «Refugiados ambientales: El nuevo desafío del Derecho Internacional del medio ambiente», Revista de Derecho, XIX, n. ${ }^{\circ}$ 2: 85-108.

Brauch, H. (2002): «Climate change, environmental stress and conflict», German Federal Ministry for ENVIRONMENT (ed.), Climate change and conflict, Berlin, Federal Ministry for Environment, Nature Conservation and Nuclear Safety, 9-112.

Diamond, J. (2005): Collapse: How societies choose to fail or survive. London: Allen Lane.

DiAmOND, L. (2008): "The democratic rollback», Foreign Affairs, 87 (2), 36-48.

Doherty, R. M. et al. (2009): «Current and future climate - and air pollution mediated impacts on human health», Environ Health, Dec. 21; 8 Suppl 1: S8.

GECHS (2009a): http://www.iss.uio.no/ gechs/gechs/

GECHS (2009b): http://www.gechs.org/ human-security/

GHF - Global Humanitarian Forum (2009): Climate Change: The anatomy of a silent crisis. http://assets. ghf-ge.org/downloads/humanimpactreport.pdf | http://www.preventionweb.net/files/9668_humanimpactreport1.pdf

GoOdman, J. (2009): «From Global Justice to Climate Justice? Justice Ecologism in an Era of Global Warming», New Political Science, 31: 4, 499-514.

HARMON, K. (2009): "Are Insurance Companies the New Climate Ally?», Scientific American, April 3.

Held, D. y Koenig-Archibugi, M. (eds.) (2005): Global Governance and Public Accountability, Blackwell: Malden, MA.

Hepburn, C. y Stern, N. (2008): «A new global deal on climate change», 
Oxford Review of Economic Policy, vol. 24(2): 259-279.

HEYD, T. (2007): Encountering Nature: Toward an Environmental Culture, Ashgate: Aldershot.

Heyd, T. y Brooks, N. (2009): «Exploring Cultural Dimensions of Adaptation to Climate Change», W. N. Adger, I. LORENZONI \& K. O'BriEN (eds.), Adapting to Climate Change: Thresholds, Values, Governance, Cambridge: Cambridge U.P., 269-282.

IKEME, J. (2003): «Equity, environmental justice and sustainability: incomplete approaches in climate change politics», Global Environmental Change, 13: 195-206.

IPCC (2007a): Climate Change 2007: Synthesis Report, Cambridge: Cambridge U.P.

IPCC (2007b): Working Group II Report: Impacts, Adaptation and Vulnerability, Cambridge: Cambridge U.P.

Keatinge, W. R. y Donaldson, G. C. (2004): "The impact of global warming on health and mortality», South Med J., nov., 97(11): 1093-9.

LEROY, S. (2006): «From natural hazard to environmental catastrophe: Past and present», Quaternary International, 158, 4-12.

LIDSKOG, R. y ELANDER, I. (2009): «Addressing Climate Change Democratically», Sustainable Development, DOI: 10.1002/sd.395.

LIDSKOG, R. y ElANDER, I. (2007): «Representation, participation or deliberation? Democratic responses to the environmental challenge», Space and Polity, 11: 75-94.

Maltais, A. (2008): «Global warming and the cosmopolitan political conception of justice», Environmental Politics, 17: 4, 592-609.

NATURE (Editorial) (2007): «The heat is on», Nature, 450, 319.
Nisbet, M. C. y Kotcher, J. E. (2009): «A Two-Step Flow of Influence? Opinion-Leader Campaigns on Climate Change», Science Communication, 30(3): 328-354.

Pengelly, L. D. et al. (2007): «Anatomy of heat waves and mortality in Toronto: lessons for public health protection», Can J Public Health, sep.-oct., 98(5): 364-8.

Pattberg, P. H. (2007): Private Institutions and Global Governance: The New Politics of Environmental Sustainability, Cheltenham: Edward Elgar.

Pew Research Center for the People AND THE PRESS (2008): «A deeper partisan divide on global warming», http://people-press.org/report/417/adeeper-partisan-divide-over-globalwarming

Pogge, Th. (2002): World Poverty and Human Rights, Cambridge, Polity Press.

Rawls, J. (1972): A theory of Justice, Harvard U.P., Cambridge, MA.

RIFKIN, J. (2002): The hydrogen economy: The creation of the worldwide energy web and the redistribution of power on earth, Cambridge: Polity and Blackwell.

Roberts, J. T. y Parks, B. C. (2007): $A$ Climate of Injustice. Global Inequality, North-South Politics, and Climate Policy, MIT Press: Cambridge, MA.

SinHA, U. K. (2010): «Climate Change and Foreign Policy: The UK Case», Strategic Analysis, 34: 3, 397-408.

SuHRKE, A. (1997): «Environmental Degradation, Migration, and the Potential for Violent Conflict», ch. 16, en Nils Petter GLeditsch et al. (eds.): Conflict and the Environment, Dordrecht: Kluwer Academic, 255-272. 
VALDivielso, J. (2004a): «¿Hay un lugar en Rawls para la cuestión ambiental?», Isegoría, n. ${ }^{\circ} 31:$ 207-220.

VALDIVIELSO, J. (2004b): «El problema de la extensión de la comunidad de justicia en el liberalismo "verde" rawlsiano», en J. RIECHMANN (ed.), Ética ecológica, Montevideo, 109128.

WISSENBURG, M. (2006): «Liberalism», en A. DOBSON and R. ECKERSLEY (eds.), Political theory and the ecolo- gical challenge, Cambridge: Cambridge U.P.

Wolf, A. T. (1999): «"Water Wars” and Water Reality: Conflict and Cooperation along International Waterways», ch. 18, en Steve Lonergan (ed.), Environmental Change, Adaptation, and Human Security, Dordrecht: Kluwer Academic, 251-265.

ZENG, N. et al. (2008): «Climate Change - the Chinese Challenge», Science, 319: 730-731.

\section{NOTAS*}

* Todos los enlaces a documentos on-line se revisaron por última vez el 8/09/2010.

${ }^{1}$ Cfr. International Climate Justice Network, «Principios Bali sobre Justicia Climática», disponible en http://www.wrm.org.uy/actores/WSSD/Bali. html.

2 Cfr. http://edition.cnn.com/2009/WORLD/europe/05/29/annan.climate.change.human/index.html

3 Definición sugerida por Thomas Heyd.

4 Cfr. http://www.wmo.int/pages/mediacentre/ news/extremeweathersequence en.html

5 Cfr. http://www.europapress.es/internacional/noticia-aumentan-muertos-incendios-sur-rusia-2010090 3185751.html
6 http://www.elmundo.es/elmundo/2010/09/03/internacional/1283507574.html

7 Cfr. http://www.pakmet.com.pk/FFD/index files/fbc.htm

8 Cfr. http://www.elpais.com/articulo/internacional/ONU/solicita/ayuda/urgente/Pakistan/elpepuint/20 100811elpepuint 4/Tes

9 http://www.noticias365.com.ve/temas/al-dia/laonu-afirma-que-amenazas-talibanes-no-frenaran-susactividades-en-pakistan-2/

10 Cfr. http://www.un.org/News/Press/docs/2007/ sc9000.doc.htm 